\title{
Zeroth-order divergence-complete discretizations of the EFIE at very low frequencies
}

\author{
E. Ubeda, J. M. Tamayo, Juan M. Rius \\ AntennaLab, Dept. of Signal Theory and Communications, \\ Universitat Politècnica de Catalunya, UPC, Jordi Girona 1-3, 08034 Barcelona (Spain) \\ E-mail: ubeda@tsc.upc.edu; jose.maria.tamayo@tsc.upc.edu; $\underline{\text { rius@tsc.upc.edu }}$
}

\begin{abstract}
We present the Self-Loop basis functions, an edge-oriented divergence-conforming set with zero charge density. These basis functions allow a rearrangement of the Linear-linear basis functions set to overcome the low-frequency breakdown of the Electric-field Integral Equation.
\end{abstract}

\section{INTRODUCTION}

Basis functions have been classified traditionally as divergence-conforming or curl-conforming. The divergence-conforming basis functions enforce normal continuity of the electric current across the edges arising in the discretization. They are wellsuited for the discretization in Method of Moments (MoM) [1] of the Electric-field Integral Equation (EFIE). The Rao, Wilton and Glisson (RWG) [2] set carries out a constant expansion of the normal component of the current across the edges. The Linear-linear (LL) set [3-5] establishes a linear expansion of the normal component of the current along the edge, which doubles the number of unknowns. Even though the Linear-linear set carries out a higher-order expansion of the current, both RWG and LL sets produce a piecewise constant expansion of the charge density.

A MoM-discretization of the EFIE with the traditional div-conforming basis functions [6] suffers from the so-called low-frequency EFIE breakdown. At very low frequencies, the contribution of the vector potential to the impedance matrix becomes negligible, according to the finite machine precision, compared with the scalar potential. This makes the discretization of the EFIE ill-conditioned and the solution inaccurate. For double machine precision and direct solution of the resulting matrix, the lowfrequency breakdown appears when analyzing objects with sizes of the mesh cells below $10^{-8} \lambda$ [7].
Divergence-conforming MoM-discretizations of the EFIE with stable condition number and accurate solution at very low frequency regime are obtained by the rearrangement of the original discretized space into the combination of a solenoidal subspace and a non-solenoidal, quasi-irrotational, subspace. The solenoidal subspace identifies the null space of the scalar potential of the EFIE and thus allows a stable discretization at very low frequencies.

The Loop-Tree and Loop-Star basis functions represent a rearrangement of the RWG-space. The Loop basis functions, expanding the RWG-solenoidal space, are vertex-oriented for simply connected surfaces and have zero divergence. They capture all the closed paths derived from the RWG discretization. Either the Tree or the Star basis functions capture the remaining non-solenoidal space, whereby they have non-zero divergence. The Tree basis functions, normally defined over planar meshings, capture all the Tree-paths in the RWG discretization. The triangle-oriented Star basis functions are normally preferred for meshing 3D objects because they are easier to implement.

There exist generalized Loop-Tree decomposition schemes for MoM-discretizations complete to higher divergence-orders [9]. However, since both RWG and LL basis functions are complete to 0 divergenceorder, such schemes are not required. In this paper, we present a new scheme to obtain a stable discretization in Method of Moments of the EFIE with the LL discretization in the very low frequency regime. Our strategy is based upon combining a novel set of basis functions, the Self-Loop basis functions, with the well-known Loop-Star basis functions. 


\section{SELF-LOOP BASIS FUNCTIONS}

The RWG and the LL basis functions are related as

$$
\vec{f}_{n}=\vec{f}_{n}^{(1)}+\vec{f}_{n}^{(2)}
$$

where $\vec{f}_{n}$ stands for the RWG basis function associated to the $\mathrm{n}$-th edge and $\vec{f}_{n}^{(1)}, \vec{f}_{n}^{(2)}$ denote, respectively, the Linear-linear basis functions of firstkind and second-kind [4] (see Fig. 1). The expression in (1) is formally equivalent to the cross-product with the normal vector of the definition in eq. 6 of [6] of the curl-conforming "Whitney forms" [6],[11]. Indeed, the RWG basis function is defined in eq. 17 in [6] as the combination of two terms that correspond to the first-kind and second-kind Linear-linear basis functions.

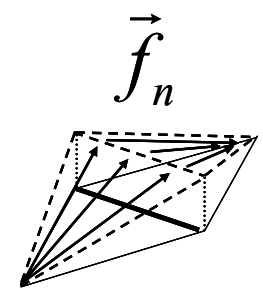

II

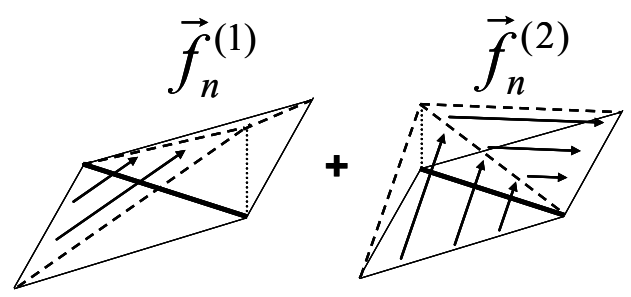

Fig. 1: Decomposition of the RWG basis functions in terms of the Firstand Second-kind Linear-linear basis functions

In view of eq. (1), we define the Self-Loop basis functions $\vec{f}_{n}^{\text {SELF }}$ as the subtraction of the first-kind and second-kind basis functions; that is,

$$
\vec{f}_{n}^{\mathrm{SELF}}=\vec{f}_{n}^{(1)}-\vec{f}_{n}^{(2)}
$$

The Linear-linear basis functions can then be expressed in terms of the RWG and the Self-Loop sets (see Fig. 2) as

$$
\vec{f}_{n}^{(1)}=\frac{1}{2}\left(\vec{f}_{n}+\vec{f}_{n}^{\mathrm{SELF}}\right)
$$

$$
\vec{f}_{n}^{(2)}=\frac{1}{2}\left(\vec{f}_{n}-\vec{f}_{n}^{\mathrm{SELF}}\right)
$$

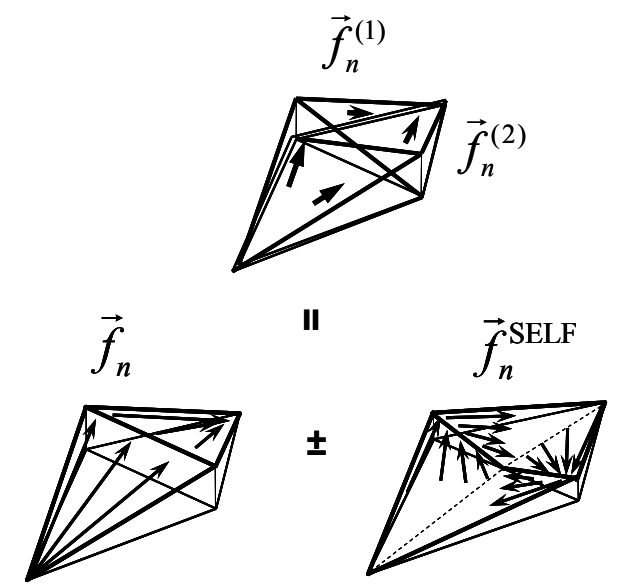

Fig. 2: LL-decomposition in terms of RWG and Self-Loop basis functions

Since the divergence of the first-kind and of the second-kind Linear-linear basis functions is the same, in view of (2), the Self-Loop basis functions have zero divergence. As shown in Fig.3, the Self-Loop basis functions are edge-oriented and ensure null charge by compensating the sign of the continuous component of the current across the edge.

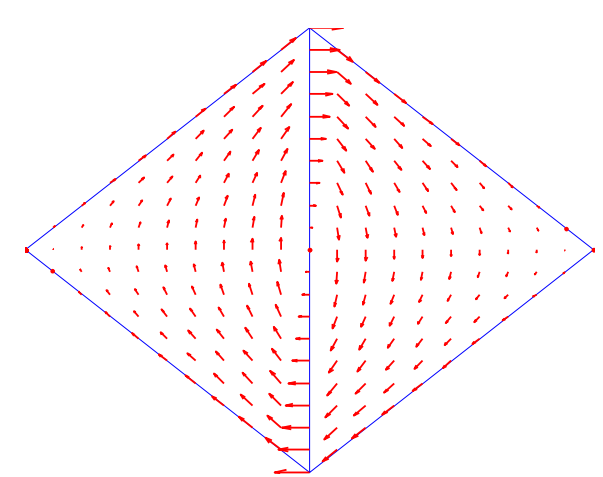

Fig. 3: Self-Loop basis functions

\section{SOLENOIDAL SPACES}

A MoM-discretization of the EFIE stable at very low-frequencies requires the complete expansion of the null-space of the scalar potential operator. This space, so-called solenoidal, requires the divergence of the current to be zero. The remaining non-solenoidal subspace of current must therefore have non zero divergence. The solenoidal subspace in the RWGdiscretized space is expanded by the vertex-oriented 
Loop basis functions $\vec{L}$. They are defined, for closed surfaces, as a linear combination of the RWG basis functions around a vertex. That is,

$$
\vec{L}_{\mathrm{v}}=\sum_{i=1}^{\mathrm{N}_{\mathrm{v}}}\left(\frac{\vec{f}_{\mathrm{v}(\mathrm{i}+1)}}{L_{\mathrm{v}(\mathrm{i}+1)}}-\frac{\vec{f}_{\mathrm{v}(\mathrm{i})}}{L_{\mathrm{v}(\mathrm{i})}}\right)
$$

where $v$ is the associated vertex-index, $\mathrm{Nv}$ stands for the number of edges around the vth-vertex and $v(i)$ denotes the edge-indices pointing the RWG basis functions involved (see Fig.4 ).

Similarly, the remaining non-solenoidal subspace is expanded by the triangle-oriented Star basis functions $\vec{S}$. They are defined, for closed surfaces, as a linear combination of the three RWG basis functions embracing the triangle as

$$
\vec{S}_{\mathrm{t}}=\frac{1}{3} \sum_{i=1}^{3} \frac{\vec{f}_{\mathrm{t}(\mathrm{i})}}{L_{\mathrm{t}(\mathrm{i})}}
$$

where $t$ stands for the associated triangle-index and $t(i)$ denotes the edge-indices of the RWG basis functions involved (see Fig. 4). As shown in eq. (5) and (6) in both definitions, the RWG-terms need to be normalized by $L$, which stands for the length of the associated edge.

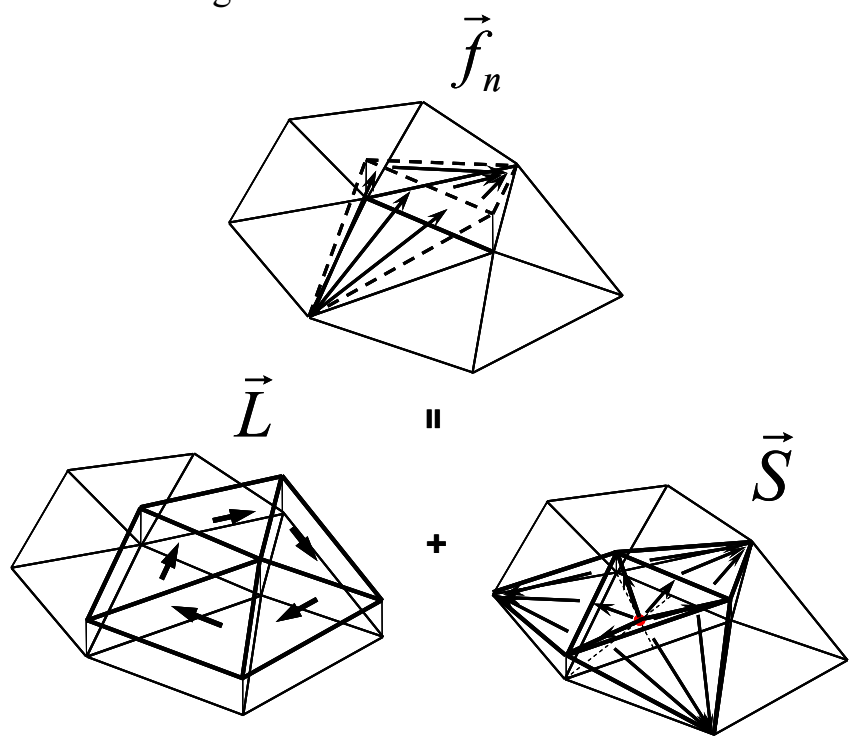

Fig. 4: RWG-decomposition in terms of Loop and Star basis functions

The solenoidal subspace in the LL-discretized space can be readily obtained from eq. (3) through the combination of the divergence-free Self-Loop basis functions and Loop basis functions. In consequence, doubling the number of unknowns in the LLdiscretized space involves increasing only the dimension of the solenoidal space of the original RWG-discretized space.

\section{IV.CONSTRUCTION OF THE IMPEDANCE MATRIX}

The impedance elements in the impedance matrices for both the [Loop;Star] and the [Loop;Selfloop;Star] sets, in view of eq. (2), (5) and (6), result from the combination of the impedance elements in the original matrices associated to EFIE[RWG] and EFIE[LL], respectively. Since for decreasing frequencies the non-solenoidal contribution becomes enhanced in the resulting [Loop;Star] or [Loop;SelfLoop;Star] matrices

$$
\left[\begin{array}{c}
E_{\text {sol }}^{\mathrm{i}} \\
E_{\text {nonsol }}^{\mathrm{i}}
\end{array}\right]=\left[\begin{array}{cc}
Z_{\text {sol-sol }}(\omega) & Z_{\text {sol-nonsol }}(\omega) \\
Z_{\text {nonsol-sol }}(\omega) & Z_{\text {nonsol-nonsol }}(1 / \omega)
\end{array}\right]\left[\begin{array}{c}
J_{\text {sol }} \\
J_{\text {nonsol }}
\end{array}\right]
$$

it is required a frequency normalization step so that the matrix becomes frequency-stable at very small frequencies [10]. If we establish as new normalized testing and expanding magnitudes

$$
\begin{aligned}
& J_{\text {nonsol }}=\omega J_{\text {nonsol }}^{\prime} \\
& E_{\mathrm{sol}}^{i^{\prime}}=\frac{E_{\mathrm{sol}}^{i}}{\omega}
\end{aligned}
$$

the previous matrix system becomes

$$
\left[\begin{array}{c}
E_{\text {sol }}^{\mathrm{i}} \\
E_{\text {nonsol }}^{\mathrm{i}}
\end{array}\right]=\left[\begin{array}{cc}
Z_{\text {sol-sol }}(1) & Z_{\text {sol-nonsol }}(\omega) \\
Z_{\text {nonsol-sol }}(\omega) & Z_{\text {nonsol-nonsol }}(1)
\end{array}\right]\left[\begin{array}{c}
J_{\text {sol }} \\
J_{\text {nonsol }}^{\prime}
\end{array}\right]
$$

\section{RESULTS}

We first analyze a cube with side $0.1 \mathrm{~m}$ discretized with 192 triangular facets under an impinging $\mathrm{x}$ polarized plane wave with + z-propagation. We show in Fig. 5 the condition number of the resulting impedance matrix for the discretizations EFIE[RWG], EFIE[LL], EFIE[Loop;Star], EFIE[Loop;Star;SelfLoop] and increasing wavelength values from $\lambda=1 \mathrm{~m}$ up to $\lambda=10^{10} \mathrm{~m}$. While EFIE[LL] and EFIE[RWG] blow up at some point, beyond the machine precision, both discretizations EFIE[Loop;Star;Self-Loop] and EFIE[Loop;Star] become stable, as expected, over the very low frequency range. We show in Fig. 6 the RCS computed with the discretizations EFIE[Loop;Star] 
and EFIE[Loop;Star;Self-loop] and $\lambda=10^{11} \mathrm{~m}$ for a sphere with radius $0.2 \mathrm{~m}$ discretized with 512 triangles under an impinging $\mathrm{x}$-polarized plane wave with $+\mathrm{z}$ propagation. Both show the same result, whereas the other discretizations EFIE[RWG] and EFIE[LL] fail. Note in Fig. 6 how the RCS-computation with EFIE[Loop;Star] and EFIE[Loop;Self-Loop;Star] gives almost the same result. This must be due to the fact that at such very low frequency regime the vector potential contribution is almost negligible with respect to the scalar potential contribution. Then, the current divergence expansion is the same for both discretizations and so must be the field they generate.

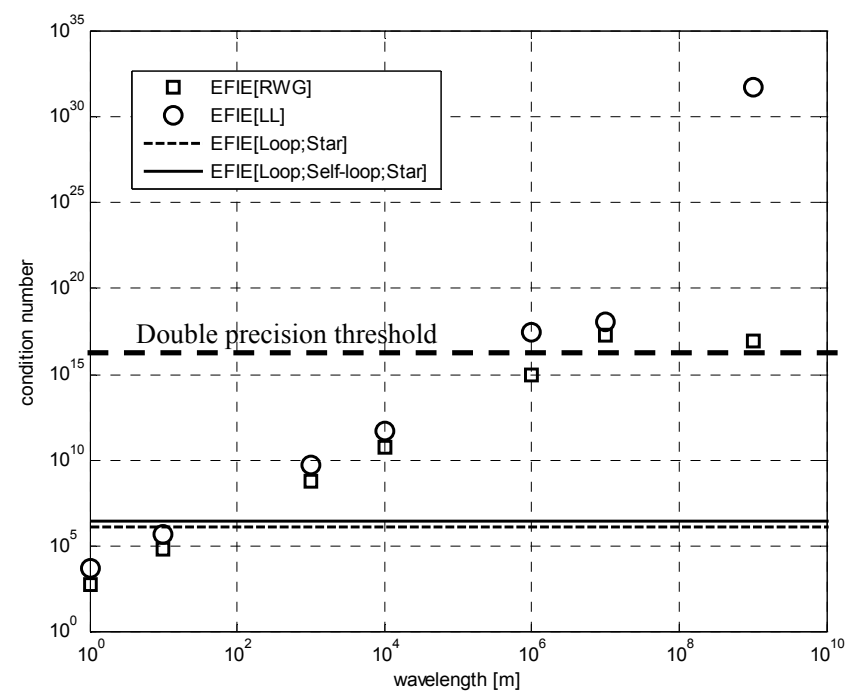

Fig. 5: Condition number for growing values of the wavelength of the impedance matrix due to the following discretizations in Method of Moments of the EFIE: [RWG], [LL], [Loop;Star] and [Loop;Star;SelfLoop]

\section{ACKNOWLEDGEMENTS}

This work was supported by the Spanish Interministerial Commission on Science and Technology (CICYT) under Projects TEC200613248-C04-02/TCM, TEC2007-66698-C04-01/TCM and CONSOLIDER CSD2008-00068 and by the "Ministerio de Educación y Ciencia" through the FPU fellowship program.

\section{REFERENCES}

[1] R. F. Harrington, "Field Computation by Moment Methods", New York: MacMillan, 1968

[2] S. M. Rao, D. R. Wilton and A. W. Glisson, "Electromagnetic Scattering by surfaces of Arbitrary Shape", IEEE Trans. Antennas Propag., vol. AP-30, No. 3, pp. 409-418, May 1982.
[3] L. C. Trintinalia and H. Ling, "First order triangular patch basis functions for electromagnetic scattering analysis", J. Electromagn. Waves Appl., 15, 1521-1537, 2001

[4] Ö. Ergül and L. Gürel, "Linear-Linear Basis functions for MLFMA solutions of magnetic-field and combined-field integral equations", IEEE Transactions on Antennas and Propagation, vol. 55, no. 4, April 2007

[5] P. Yla-Ölijala, M. Taskinen and S. Järvenpää, "Surface integral equation formulations for solving electromagnetic scattering methods with iterative methods", Radio Science, vol. 40, RS6002, doi: 10.1029/2004RS003169, 2005

[6] R. D. Graglia, D. R. Wilton and A. F. Peterson, "Higher Order Interpolatory Vector Bases for Computational Electromagnetics", IEEE Trans. on Antennas and Propag., vol. 45, No. 3, pp. 329-342, March 1997.

[7] Z. G. Qian and W. C. Chew, "A quantitative study on the low frequency breakdown of the EFIE", Microwave and Optical Technology letters, vol. 50, no. 5, May 2008.

[8] W. Wu, A. W. Glisson amd D. Kajfez, "A study of two numerical procedures for the Electric Field Integral Equation at low frequency", Appl. Computat. Electromagn. Soc. J., vol. 10, no. 3, November 1995.

[9] R. A. Wildman and D. S. Weile, "An accurate Broad-band method of moments using higher basis functions and Tree-Loop decomposition", IEEE Transactions on Antennas and Propagation, vol. 52, no. 11, November 2004

[10] J. Zhao and W. C. Chew, "Integral equation solution of Maxwell's equations from 0 frequency to microwave frequencies", IEEE Transactions on Antennas and Propagation, vol. 48, no. 10, pp. 16351645, October 2000

[11] A. Bossavit, "Whitney forms: A class of finite elements for threedimensional computations in electromagnetics", Inst. Elect. Eng. Proc., vol. 135 , pt. A, no. 8, pp. 493-500, 1988

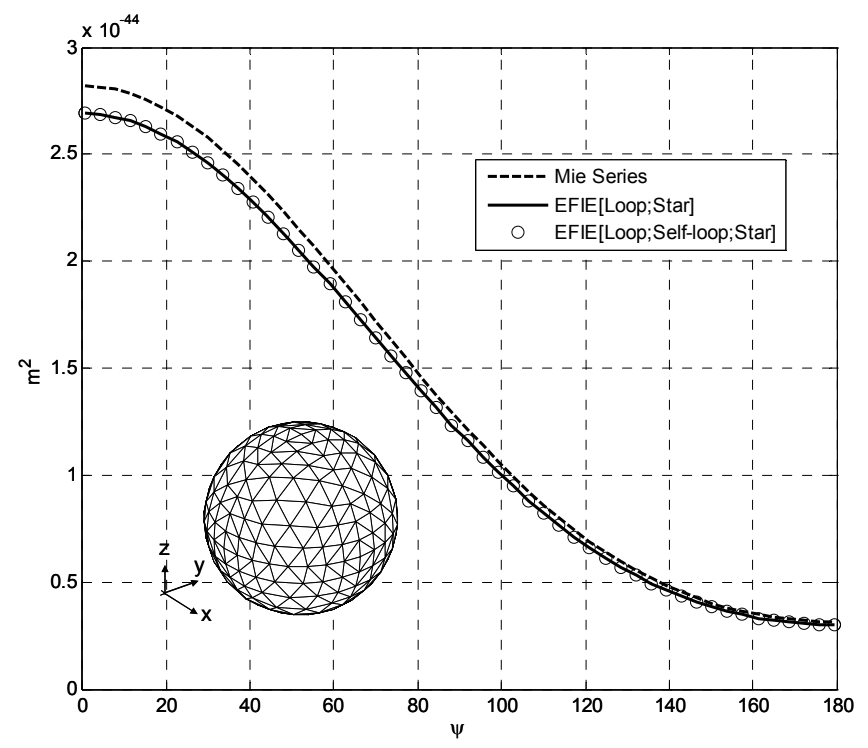

Fig. 6: Bistatic yz-plane for a sphere with radius $=0.2 \mathrm{~m}$ meshed with 512 triangles under an impinging plane wave with $\lambda=10^{11} \mathrm{~m}$ 